\title{
An Efficient Structure of Organization with Complete Group Guidance
}

\author{
Satoshi Ikeda ${ }^{1, *}$, Makoto Sakamoto $^{1}$, Takao Ito ${ }^{2}$ \\ ${ }^{1}$ Department of Computer Science and System Engineering, Miyazaki University, 1-1 Gakuen Kibanadai Nishi, Miyazaki, 889-2192, Japan \\ ${ }^{2}$ Graduate School of Engineering, Hiroshima University, Higashi-Hiroshima, 739-8526, Japan
}

\section{ARTICLE INFO}

\section{Article History}

Received 23 October 2018

Accepted 23 November 2018

Keywords

Hierarchical organization mathematical model

discrete combination optimization

\begin{abstract}
Organizational structure is one of the most important factors in determining its performance. Our interest is what kind of structures is efficient, and what conditions are required for an efficient structure? In our previous papers, we proposed a mathematical model for evaluating the hierarchical organization quantitatively and used it to investigate this problem. As a result of that research, we proved that the shape of the hierarchical organization that maximizes the evaluation value of organization, can be classified into three types depending on the capacity value of the members when number of the evaluation criteria is only one. The purpose of this paper is to extend previously obtained results to an improved mathematical model.
\end{abstract}

(C) 2019 The Authors. Published by Atlantis Press SARL.

This is an open access article distributed under the CC BY-NC 4.0 license (http://creativecommons.org/licenses/by-nc/4.0/).

\section{INTRODUCTION}

There are many criteria for evaluating members of an organization. For example, technical skills, management skills, etc. In Ikeda [1] and Ikeda et al. [2], we proved by using a mathematical model that an efficient shape of a hierarchical organization with only one criterion is limited to three types (Figure 1).

Subsequently, the mathematical model for evaluating the shape of the organization has been generalized more realistically $[3,4]$. Although the basic idea of the total output to the outside of the organization as an evaluation value has not changed, the major improvements in the revised mathematical model are the following two points.

(a) Contribution of the members for a given organization can be categorized as the internal contribution to maintain the organization and the external contribution to realize the purpose of the organization in society.

(b) For any fixed organization, the sum of the effort each person spends is constant, but its influence is different depending on how to take a communication style.

The ratio of one's external contribution effort to the total of one's effort is called the external output coefficient. In the mathematical model of Ikeda et al. [3,4] with one evaluation criterion under the assumption that the external output coefficient takes a constant $\alpha \in[0,1]$ independent of member, previous results in Ikeda [1] and Ikeda et al. [2] correspond to $\alpha=0$ and the communication style called "the complete group guidance".
When the number of evaluation criteria is one, in the paper Ikeda et al. [3,4], we showed that the structure of an efficient organization becomes line shape (Figure 1b), in the case that personal ability value of organization members is high. This is an extension of the results of Ikeda [1] and Ikeda et al. [2] to the case of the model of Ikeda et al. [3,4] in part. However, in general it was not known whether the results of Ikeda [1] and Ikeda et al. [2] (Figure 1c), could be extended to the case of the model of Ikeda et al. [3,4]. We expected that the results could be extended in the general case, but in reality it was not. Yoshimura et al. [5] by simulation experiments showed that an efficient tree is generally a brush tree shape (Figure 2), when the number of evaluation criteria is one. After much consideration, we concluded that the efficient tree shape as shown in Figure 1 may depend on the communication style.

The purpose of this paper is to show that the efficient organizational shape when the evaluation criteria is only one, is limited to the three types shown in Figure 1 under the assumption of the communication style called the complete group guidance.

\section{MATHEMATICAL MODEL}

Now we will introduce a mathematical model for evaluating the hierarchical organization quantitatively $[3,4]$.

\subsection{Notations and Tree Graph as Hierarchical Organization}

Suppose that $G=(V(G), E(G))$ is a graph. Throughout this paper, a graph is always finite, directed and simple, with order $n=|V(G)|$ $(n \geqq 2)$ and size $m=|E(G)|$. 


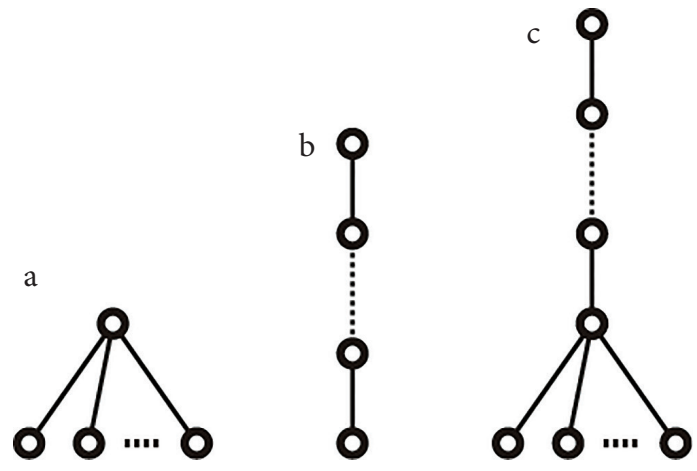

Figure $1 \mid$ Three types of efficient trees when the number of the evaluation criteria is one.

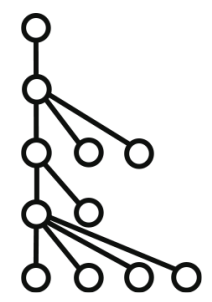

Figure 2 A brush graph.

For $u \in V(G)$, by $N(u)=\{v \mid\{u, v\} \in E(G)\}$, we denote the set of vertices adjacent to $u$, and call $\operatorname{deg}(u)=|N(u)|$ the degree of $u \in V(G)$. For an arbitrarily fixed rooted tree $T$ with root $r$ and its leaves set $L(T)$, we denote

$$
\operatorname{deg}^{\star} s= \begin{cases}\operatorname{deg} s & \text { if } s \in\{r\} \cup L(T), \\ \operatorname{deg} s-1 & \text { otherwise }\end{cases}
$$

For a rooted tree $T$ with $V(T)=S$, we define natural partial ordering $\$(S,<) \$$ that so-called the tree-order associated with $T$. That is, the root $r \in S$ of $T$ is the least element and the leaves of $T$ are the maximal elements in this partial order. This ordering will be considered as the expression "depth": if $x<y$, we say $x$ lies below $y$ in $T$. Note that the root $r$ is the least element, and that the leaves of $T$ are its maximal elements in this partial order.

Suppose that $S=\left\{s_{1}, s_{2}, \ldots, s_{n}\right\}(n \geq 2)$ and $\mathcal{A}(|\mathcal{A}| \geqq 1)$ are finite sets. Through this paper, $\mathcal{A}$ is the set of the evaluation measures. And $S$ is interpreted as the set of members of a given organization, which consists of $s_{1}, s_{2}, \ldots, s_{n}$. There are many rooted trees with $S$ as vertices. After that, we identify each rooted tree as its corresponding organization tree. Let $\mathcal{T}(S)$ be the set of rooted trees with $S$ as its elements.

\subsection{External Contribution and Internal Contribution}

For a given $S$ and $i \in \mathcal{A}$, we define $\left\{\varphi_{i}\right\}_{i \in \mathcal{A}}$ by

$$
\varphi_{i}: S \rightarrow R^{+} \equiv\{x \in R \mid x>0\} \quad \text { for } i \in \mathcal{A}
$$

We call $\varphi_{i}(s)$ the personal ability of $s \in S$ with respect to $i \in \mathcal{A}$. And we call $\left(S,\left\{\varphi_{i}\right\}_{i \in \mathcal{A}}\right)$ an evaluation system. According to the idea of 1-3, the evaluation function of a given evaluation system 1 $\left(S,\left\{\varphi_{i}\right\}_{i \in \mathcal{A}}\right)$ is defined as follows. For $i \in \mathcal{A}$, total output of $s \in S$ that written by $f_{s \rightarrow}^{\alpha, i}$ is the product of $s^{\prime}$ s personal ability $\varphi_{i}(s)$ and $s$ 's input that written by $f_{s \leftarrow}^{\alpha, i}$. That is,

$$
f_{s \rightarrow}^{\alpha, i}=\varphi_{i}(s) f_{s \leftarrow}^{\alpha, i} .
$$

For $i \in \mathcal{A}$, s's total output $f_{s \rightarrow}^{\alpha, i}$ is classified into $s^{\prime}$ s external output $f_{s \rightarrow \text { out }}^{\alpha, i}$ and $s^{\prime}$ s internal output $f_{s \rightarrow \text { in }}^{\alpha, i}$ (Figure 3). That is,

$$
f_{s \rightarrow}^{\alpha, i}=f_{s \rightarrow \text { out }}^{\alpha, i}+f_{s \rightarrow \text { in }}^{\alpha, i} .
$$

For the subordinate $s \in S$ who received instructions from his superior, it is necessary to transmit appropriate instructions $f_{s \rightarrow \text { in }}^{\alpha, i}$ as superior to his own subordinates, while $s$ as subordinate carries out the instructions $f_{s \rightarrow \text { out }}^{\alpha, i}$ (Figure 3).

We assume that the ratio of $s^{\prime}$ s external output to $s^{\prime}$ s total output is a constant independent of $s \in S$. Through this paper, we denote the ratio of $f_{s \rightarrow \text { out }}^{\alpha, i}$ to $f_{s \rightarrow}^{\alpha, i}$ as $\alpha \in[0,1]$ for any $s \in S$. That is,

$$
\begin{array}{ll}
\text { external output } f_{s \rightarrow \text { out }}^{\alpha, i}: & \text { internal output } f_{s \rightarrow \text { in }}^{\alpha, i} \\
= & \alpha
\end{array}
$$

for any $s \in S$. We call $\alpha$ the external output coefficient and call $1-\alpha$ the internal output coefficient.

\subsection{Weights' Policy}

For a given organizational structure tree $T$, we assume that the value of the input for subordinate $x \in N(s)$ with $x>s$ is obtained by multiplying its weight $\left\{w_{s x}^{i}\right\}$ to $f_{s \rightarrow \text { in }}^{\alpha, i}$ (see Figure 3 again). Where

$$
0 \leq w_{s x}^{i} \leq 1
$$

for any $s \in S \backslash L(T), x>s$ of $x \in N(s)$ and

$$
1 \leq \sum_{x \in N(s), x>s} w_{s x}^{i} \leq \operatorname{deg}{ }^{*} s
$$

for any $s \in S \backslash L(T)$. Therefore, the total contribution of $s \in S \backslash L(T)$ for the organization can be expressed by

$$
\left(\sum_{x \in N(s), x>s} w_{s x}^{i}\right) f_{s \rightarrow \text { in }}^{\alpha, i}+f_{s \rightarrow \text { out }}^{\alpha, i} .
$$

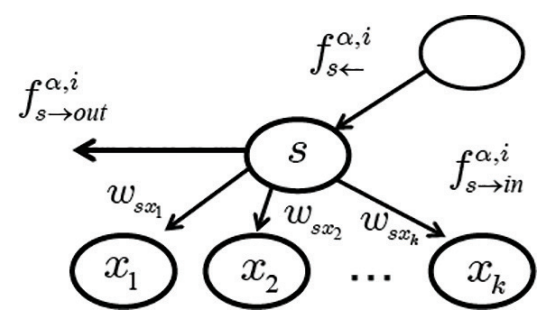

Figure 3 Input and output for $s \in S$. 
We assume that $\left(w_{s x}^{i}\right)_{x \in N(s), x>s}$ for $s \in S \backslash L(T)$ is a sequence depending only on $\operatorname{deg}^{*} s$ and $i \in \mathcal{A}$. That is, for $s, s^{\prime} \in S \backslash L(T) s, s^{\prime}$ and $i \in \mathcal{A}, \operatorname{deg}^{*} s=\operatorname{deg}^{*} s^{\prime}$ implies that $\left(w_{s^{\prime} x}^{i}\right)_{x \in N\left(s^{\prime}\right), x>s^{\prime}}$ is a permutation of $\left(w_{s x}^{i}\right)_{x \in N(s), x>s}$. Thus, for a fixed $\operatorname{deg}^{*} s$ and $i \in \mathcal{A}$, the selection that we can do is which weight to assign whom. We call the way of the determination a weights' policy. For any weights' policy, we assume that if $\operatorname{deg}^{*} s \geqq \operatorname{deg}^{*} s^{\prime}$ for $s, s^{\prime} \in S$,

$$
w_{s x 1}^{i} \geq w_{s x 2}^{i} \geq \cdots \text { and } w_{s^{\prime} x^{\prime} 1}^{i} \geq w_{s^{\prime} x^{\prime} 2}^{i} \geq \cdots
$$

then $\left(w_{s x}^{i}\right)_{x \in N(s), x>s}$ and $\left(w_{s^{\prime} x^{\prime}}^{i}\right)_{x^{\prime} \in N\left(s^{\prime}\right), x^{\prime}>s^{\prime}}$ satisfy

$$
w_{s x j}^{i} \leq w_{s^{\prime} x j}^{i}
$$

for $j=1,2, \ldots, \operatorname{deg}^{*} s^{\prime}$.

\subsection{Communication Style}

The weights' policy is a parameter determined by the communication style in the organization. Typical ones are the following two cases;

(1) Complete Individual Guidance (CIG)

$$
\sum_{x \in N(s), x>s} w_{s x}^{i}=1 \text { for any } s \in S \backslash L(T) .
$$

(2) Complete Group Guidance (CGG)

$$
\sum_{x \in N(s), x>s} w_{s x}^{i}=\operatorname{deg}^{\star} s \text { for any } s \in S \backslash L(T) .
$$

The case of (1) CIG is an organization like a secret society. Since a superior gives an instruction to his/her subordinates individually, the members of the organization do not know about each other's work. In such organizations, when new member joins the organization, or a member of the organization leave the organization, its efficient organization cannot be constructed unless the existing organization is broken once.

On the other hand, the case of (2) CGG is an open organization. The characteristic of an organization with such a communication style is that it is stable in the sense that there is no need to significantly change the composition of the whole organization when new member joins it. Roughly speaking, the reason why CGG has such a property is that when we convert $T$ to

$$
T^{\prime}=T-x s+y s
$$

for $T, T^{\prime} \in \mathcal{T}(S), s, x, y \in S, s \in N(x), s>x$, the evaluation function is affected only by the part of the up closure of $s$ (Figure 4).

CIG and CGG are extreme cases, the general style is intermediate between them.

\subsection{Evaluation Function}

To summarize so far, for a given evaluation system $\left(S,\left\{\varphi_{i}\right\}_{i \in \mathcal{A}}\right)$, a rooted tree $T$ with root $r$ and $V(T)=S, s \in S, i \in \mathcal{A}$ and an external

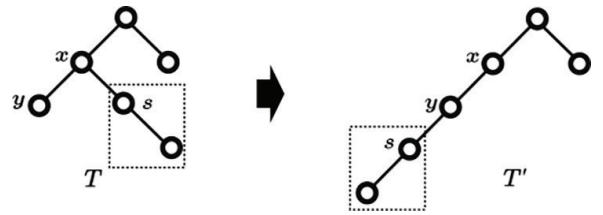

Figure 4 Conversion from $T$ to $T^{\prime}$.

output coefficient $\alpha \in[0,1], f_{s \leftarrow}^{\alpha, i}$ and $f_{s \rightarrow}^{\alpha, i}$ denote the input and the output of $s \in S$, respectively. And $f_{s \rightarrow \text { out }}^{\alpha, i}$ and $f_{s \rightarrow \text { in }}^{\alpha, i}$ denote the external and the internal outputs of $s \in S$ for the organization, respectively. Then, we define as follows:

$$
\begin{aligned}
f_{s \rightarrow}^{\alpha, i} & =\varphi_{i}(s) f_{s \leftarrow}^{\alpha, i} \\
f_{s \leftarrow}^{\alpha, i} & = \begin{cases}1 & \text { if } s=\text { root, } \\
w_{p(s) s}^{i} f_{p(s) \rightarrow \text { in }}^{\alpha, i} & \text { otherwise. }\end{cases}
\end{aligned}
$$

Where $\left\{w_{x y}^{i}\right\}$ denote the weights from $x \in S \backslash L(T)$ to $y \in N(x)$ with respect to $i \in \mathcal{A}$ in an arbitrarily fixed $T$. And $p(s)$ denotes the parent node (as superior) of $x \in S$ on $T$. Under the assumption of external and internal output, we define

$$
\begin{aligned}
f_{s \rightarrow \text { in }}^{\alpha, i} & = \begin{cases}0 & \text { if } s \in L(T), \\
(1-\alpha) f_{s \rightarrow}^{\alpha, i} & \text { otherwise, }\end{cases} \\
f_{s \rightarrow \text { out }}^{\alpha, i} & = \begin{cases}f_{s \rightarrow}^{\alpha, i} & \text { if } s \in L(T), \\
\alpha f_{s \rightarrow}^{\alpha, i} & \text { otherwise. }\end{cases} \\
f_{s \rightarrow}^{\alpha, i} & =f_{p(s) \rightarrow \text { in }}^{\alpha, i}+f_{p(s) \rightarrow \text { out }}^{\alpha, i},
\end{aligned}
$$

For a given evaluation system $\left(S,\left\{\varphi_{i}\right\}_{i \in \mathcal{A}}\right)$ an external output coefficient $\alpha \in[0,1]$ and a given weights' policy, let $T$ be a rooted tree graph with $V(T)=S$. Then, we will evaluate the rooted tree $T$ as organization model by

$$
\Phi^{(\alpha)}(T)=\sum_{i \in \mathcal{A}} \Phi^{(\alpha, i)}(T), \Phi^{(\alpha, i)}(T)=\max _{\left\{w_{x y}^{i}\right\}} \sum_{i \in \mathcal{A}} f_{s \rightarrow \text { out }}^{(\alpha, i)}(T)
$$

Here $\left\{w_{x y}^{i}\right\}$ shall be taken about all the possible combinations under the given weights' policy. For a given $\left(S,\left\{\varphi_{i}\right\}_{i \in \mathcal{A}}\right)$ and $\alpha \in[0,1]$, we call $\Phi^{(\alpha)}(T)$ the ability value of $T$. Under a given weights' policy, $T_{\text {opt }}^{(\alpha)}$ is an efficient tree for a given external output coefficient $\alpha \in[0,1]$, if $\max _{T \in \mathcal{T}(S)} \Phi^{(\alpha, i)}(T)$ is attained by $\Phi^{(\alpha)}\left(T_{\mathrm{opt}}^{(\alpha)}\right)$. Here $\mathcal{T}(S)$ denotes the set of rooted tree graph with $V(T)=S$.

\section{RESULTS}

Theorem 1. Under the setting of Complete Group Guidance, suppose that $\left(S,\left\{\varphi_{i}\right\}_{i \in \mathcal{A}}\right)$ is an evaluation system such that $S=\left\{s_{1}, s_{2}, \ldots, s_{n}\right\}$ with

$$
\varphi_{i}\left(s_{1}\right) \geq \varphi_{i}\left(s_{2}\right) \geq \cdots \geq \varphi_{i}\left(s_{n}\right)
$$

for some $i \in \mathcal{A}$. Assume that $\max _{T \in \mathcal{T}(S)} \Phi^{(\alpha, i)}(T)$ is attained by $T_{\text {opt }}^{(\alpha, i)}$, then we have the following.

(a) Assume that $\varphi_{i}\left(s_{n}\right) \geq 2 /(1-\alpha)$, then we see that one of $T_{\text {opt }}^{(\alpha, i)}$ is the path graph in Figure 5 (1).

(b) Assume that $\varphi_{i}\left(s_{2}\right) \leq 1 /(1-\alpha)$, then we see that one of $T_{\mathrm{opt}}^{(\alpha, i)}$ is the star graph in Figure 5 (2). 


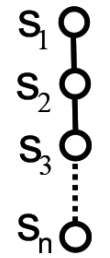

(1) $T_{o p t}^{(\alpha, i)}$ as a path graph (2) $T_{o p t}^{(\alpha, i)}$ as a star graph

Figure 5 An efficient organization structure.

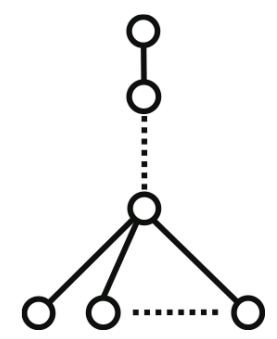

Figure 6 General form of $T_{\mathrm{opt}}^{(\alpha, i)}$ for some $i \in \mathcal{A}$.

The typical form of an efficient tree with respect to some $i \in \mathcal{A}$ is a path graph or star graph as in Theorem 1. In general, we can show that the form of $T_{\text {opt }}^{(\alpha, i)}$ becomes the form which seems to have put these two figures together.

For a given $\left(S,\left\{\varphi_{i}\right\}_{i \in \mathcal{A}}\right)$ and its organizational structure tree $T$, let us define

$$
D_{2}(T)=\left\{s \in S \mid \operatorname{deg}{ }^{\star}(s) \geq 2\right\}
$$

Theorem 2. Under the setting of CGG, suppose that $\left(S,\left\{\varphi_{i}\right\}_{i \in \mathcal{A}}\right)$ is an evaluation system. Assume that $\max _{T \in \mathcal{T}(S)} \Phi^{(\alpha, i)}(T)$ is attained by $T_{\text {opt }}^{(\alpha, i)}$ for some $i \in \mathcal{A}$, then we have the following.

(a) $\left|D_{2}\left(T_{\text {opt }}^{(\alpha, i)}\right)\right|$ is equal to 0 or 1.

(b) Putting $D_{2}\left(T_{\text {opt }}^{(\alpha, i)}\right)=\{x\}$ when $\left|D_{2}\left(T_{\text {opt }}^{(\alpha, i)}\right)\right|=1$, then we see that

$$
\left\{s \in S \mid s>x \text { in } T_{\mathrm{opt}}^{(\alpha, i)}\right\}=L\left(T_{\mathrm{opt}}^{(\alpha, i)}\right) .
$$

The general form of the most efficient tree $T_{\text {opt }}^{(\alpha, i)}$ which Theorem 2 insists on is shown in Figure 6.

\section{CONCLUSION}

In this paper, we introduced that the conclusions of the papers [1,2] are also valid under a new mathematical model of the organization $[3,4]$. The details of the proof are omitted, but they can be shown in the same way as the papers $[1,2]$. Eventually, even in the improved new mathematical model, we found that the efficient organiza-
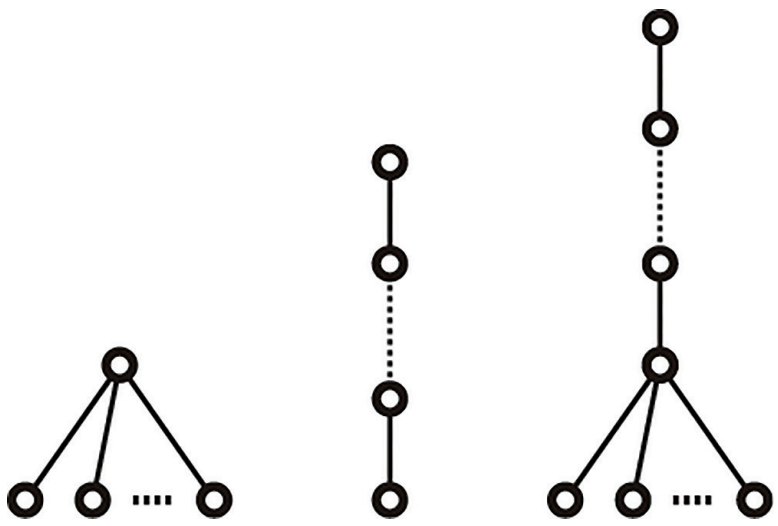

Figure 7 Three types of efficient trees.

tional shape can be classified into the three types shown in Figure 7 , whenever the organizational communication style is CGG and there is only one organizational evaluation.

However, Yoshimura et al. have experimentally shown that there are efficient organizational shape other than these three in the general communication style. The theoretical analysis of the general case remains an open problem.

\section{CONFLICTS OF INTEREST}

The authors declare they have no conflicts of interest.

\section{ACKNOWLEDGMENTS}

The authors are much indebted to M. Yoshimura for his invaluable comments.

\section{REFERENCES}

[1] S. Ikeda, T. Ito, M. Sakamoto, An efficient structure of organization, International Interdisciplinary Workshop on Robotics, Ecosystem, and Management, Yamaguchi, Japan, 2009, pp. 72-78.

[2] S. Ikeda, T. Ito, M. Sakamoto, Discovering the efficient organization structure: horizontal versus vertical. Artif. Life Robot. 15 (2011), 478-481.

[3] S. Ikeda, T. Ito, M. Sakamoto, A mathematical model of hierarchical organization. International Conference on Pure Mathematics and Applied Mathematics (PM-AM2015), Vienna, Austria, 2015 pp. 423-432.

[4] S. Ikeda, T. Ito, M. Sakamoto, T. Ito, An evaluation model of internal and external contributions in hierarchical organizations, Int. J. Math. Mod. Methods Appl. Sci. 9 (2015), 636-645.

[5] M. Yoshimura, T. Ito, M. Sakamoto, T. Ito, Y. Shinano, S. Ikeda, An efficient structure of organization with complete individual guidance. Proceedings of the 2018 International Conference on Artificial Life and Robotics (ICAROB2018), Beppu, Oita, Japan, 2018, pp. 264-267. 


\section{Authors Introduction}

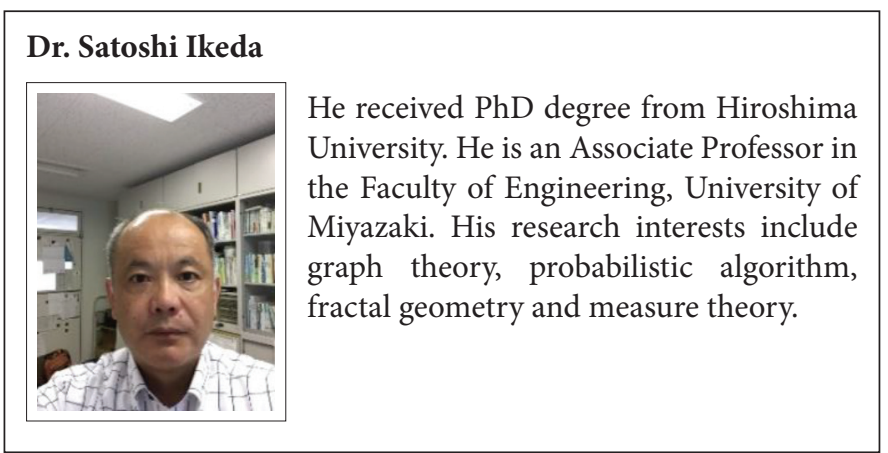

Dr. Makoto Sakamoto

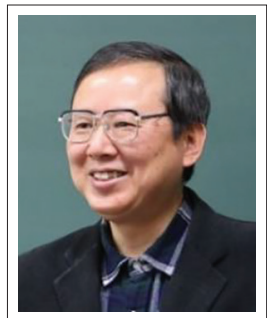

He received the $\mathrm{PhD}$ degree in Computer Science and Systems Engineering from Yamaguchi University in 1999. He is presently an Associate Professor in the Faculty of Engineering, University of Miyazaki. His first interests lay in hydrodynamics and time series analysis, especially the directional wave spectrum. His current main research interests are automata theory, languages and computation.

\section{Dr. Takao Ito}

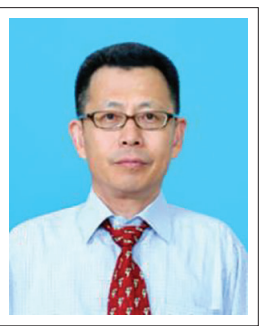

$\mathrm{He}$ is Professor of Management of Technology (MOT) in Graduate School of Engineering at Hiroshima University. He is serving concurrently as Professor of Harbin Institute of Technology (Weihai) China. He has published numerous papers in refereed journals and proceedings, particularly in the area of management science, and computer science. He has published more than eight academic books including a book on Network Organizations and Information (Japanese Edition). His current research interests include automata theory, artificial intelligence, systems control, quantitative analysis of inter-firm relationships using graph theory, and engineering approach of organizational structures using complex systems theory. 